\title{
Who is willing to pay for science? On the relationship between public perception of science and the attitude to public funding of science
}

\author{
Ana Muñoz, Carolina Moreno and José Luis Luján
}

This article examines the relationship between the general public's understanding of science and the attitude towards public funding of scientific research. It applies a multivariate and discriminant analysis (Wilks' Lambda), in addition to a more commonly used bivariate analysis (Cramer's V), to data compiled from the Third National Survey on the Social Perception of Science and Technology in Spain (FECYT, 2006). The general conclusion is that the multivariate analysis produces information complementary to the bivariate analysis, and that the variables commonly applied in public perception studies have limited predictive value with respect to the attitude towards public funding of scientific research.

Keywords: public attitude to science, public funding of science, public perception of science

\section{Introduction}

Public perception studies have evolved amidst the consolidation of public policies for funding scientific research. They were originally fostered by the United States National Science Foundation (NSF) with the aim of establishing an indicator of the public understanding of science. Through OECD initiatives, the science policy model of the NSF spread over time to other countries and with it, science and technology measurement (Godin, 2002), including indicators concerned with the public perception of science and technology.

One of the main objectives of studies on the public perception of science has been the creation of an indicator of the social support for public policies aimed at promoting scientific research (Kallerud and Ramberg, 2002; Miller, 2004). The underlying premise is that the degree of knowledge has a considerable bearing on interest in science and its perception.

Generally, the implicit interest of studies of public perception is to determine the general attitudes towards science as those are considered useful in predicting overall attitudes towards most public science and technology policies. Consequently, questionnaires in studies on the public perception of science traditionally pose the questions drawn from the following themes: 
Interest. A set of questions aimed at determining the degree of interest in scientific and technological issues in comparison with other topics (such as politics, art, economics, sport, health, the environment, etc.). At times, this may also involve examining public interest in different fields of science and technology (computer science, nuclear energy, biotechnology, space exploration, etc.).

Knowledge. Through a series of questions, the degree of scientific knowledge of the persons interviewed is gauged. Some studies have sought to determine the public's familiarity with "scientific methodology."

The general understanding of science and technology. This theme has basically consisted of securing the general public perception of scientific research (with few references to technology, except in specific studies). As we have pointed out, the main objective of these questions has been to establish an indicator of public support for scientific research (interpretable as support for public funding).

For some time now, public perception studies have undergone numerous instances of critical analysis. The main criticisms are the following:

Technical aspects. This kind of criticism has mainly focused on questionnaire design and the scales that have been used. Some authors have suggested ameliorating the measurement instruments in order to improve the quality of empirical information (Allum et al., 2008; Pardo and Calvo, 2002, 2004).

The concept of science. It has been suggested that public perception questionnaires make reference to a concept of science that is out of context. In general, following on from this line of argument, emphasis is placed on the need to carry out qualitative studies in situations that involve the public's genuine interaction with science and technology (Irwin and Michael, 2003; Michael, 1992, 2002; Millar and Wynne, 1988).

The concept of scientific culture. This criticism has focused on the definition of scientific culture taken for granted in public perception studies. There is a debate on whether quantitative studies of public perception presuppose a fixed definition of scientific culture or whether they focus on the scientifically informed individual. More specifically, criticism is levelled at the idea of "scientific literacy" (López Cerezo and Cámara, 2007; Millar and Wynne, 1988).

The cognitive deficit model. Criticism is geared at the principal explanatory hypothesis, namely that perception depends mainly on knowledge. It is undoubtedly the most widespread critical argument in the literature. It maintains that regardless of whether quantitative or qualitative studies are used, it is necessary to explore other determinants of public perception of science and technology (Irwin and Wynne, 1996; Millar and Wynne, 1988; Sturgis and Allum, 2004).

The very concept of the perception of science (and technology). This line of criticism questions the very concept of the perception of science. Its significance as well as its validity and usefulness are questioned in terms of providing an indicator of a given attitude towards scientific knowledge, its possible applications and its public funding (Allum et al., 2008; Bauer, 2002; Luján and Todt, 2000; Michael, 1992).

Our paper will focus on the last issue. Even though the scope is quite broad, there are two questions of particular relevance: 1) the relationship between the perception of science in general and the perception of research in specific fields or related to specific technological applications (for instance, biotechnology or food safety: Bauer, 2002; Luján and Todt, 2000; Todt et al., 2007, 2009); 2) the usefulness of the general perception or appraisal of science as 
an indicator of the social support for public funding policies on scientific research. Both questions are related. In fact, the implicit objective of studies on the public perception of science and technology would entail generating a kind of "general factor" that is indicative for the overall appraisal of science. This general factor would depend on the degree of knowledge and would be linked to the support given to both specific technological developments and the public funding of scientific research.

Of the two issues, the one related to funding is the specific objective of this study. We start from the hypothesis that support for public funding of scientific research is an indicator of a general attitude to science; therefore, the study is intended to identify those features that best characterize those subjects who are in favour of public expenditure for scientific research as compared to those who are not.

\section{Methodology}

\section{Survey}

Data from the Third National Survey on the Social Perception of Science and Technology (2006) were used; the sample size was 6,998 persons, selected from the Spanish population and aged 15 or above. The questionnaire used contained questions that addressed the following areas: Interest and information; Perception and attitudes; The social appropriation of science and technology; Science and technology policy. The specific questions related to those areas, overall, are coherent with other science and technology perception studies, and measure a mix of knowledge, perceptions, beliefs and attitudes.

To identify the relationship between the variables that measure general perception of science and the attitude towards the public funding of scientific research, the question selected as the dependent variable was the one identifying support for public funding in different policy sectors. Specifically, the question was: "Imagine you could choose the use of public fundings. I will show you a card with different policy sectors. Please, tell me in which of them you would increase public expenditure" (up to three sectors). The answer options included the following areas: public works, public security and defence, transport, science and technology, the environment, justice, culture and sports. A dichotomous variable was created: one value corresponding to the interviewees who selected the option "science and technology," regardless of whether they chose it as a first, second or third option; the other corresponding to persons who did not choose this area at all. In accordance with the results, 1,388 (19.7\%) answers indicate that interviewees would invest in scientific research if they had the opportunity to allocate public financial resources, while 5,666 (80.3\%) would not; that is to say most of the subjects did not chose this sector at all. ${ }^{1}$

As independent variables, the questions were selected in order to best reflect the general perception of science. The questions are the following: ${ }^{2}$

- Interest in different topics (scale from 1 to 5). The topics were: food and consumption; science and technology; film, art and sculpture; sports; economics and the business world; medicine and health; environment and ecology; politics; and, celebrities.

- Evaluation of professions (scale from 1 to 5): doctors, scientists, engineers, judges, lawyers, sportsmen and sportswomen, artists, journalists, businesspeople, teachers and university professors, priests and politicians.

- The level of agreement with statements on different aspects of scientific knowledge and technological development (scale from 1 to 5). The statements were the following: 1) we give too much value to scientific and technological knowledge in comparison with other 
forms of knowledge, 2) science and technology provide the best and most reliable knowledge about the world, 3) scientific research and technology will help to cure illnesses such as AIDS, cancer, etc., 4) science and technology applications have created health risks, 5) science and technology applications are leading to the loss of jobs, 6) thanks to science and technology, there will be more job opportunities for future generations, 7) science and technology applications are creating an artificial and inhuman lifestyle, 8) science and technology are making life easier and more comfortable for us, 9) science and technology will help to get rid of poverty and hunger in the world, 10) science and technology are widening the gap between rich and poor countries, 11) science and technology contribute to improve the natural environment, 12) science and technology applications are creating serious environmental problems, 13) science and technology are not concerned about true social needs, 14) science and technology enable social welfare to be improved.

- The balance between positive and negative aspects of science and technology. The interviewees had to choose one of the following options: 1) overall, the benefits of science and technology are greater than any harmful effects; 2) overall, benefits and harmful effects of science and technology are balanced; 3) overall, harmful effects of science and technology are greater than any benefits.

- The relationship between science and technology. The interviewees had to choose one of the following options: 1) science and technology are, in every respect, the same; 2) science and technology are, to some extent, the same; 3) science and technology are not exactly the same; 4) science and technology are not the same; 5) I have not a position on this issue.

- Concepts associated with science and (in a different question) technology (scale from 1, no association, to 5, strong association): progress, dehumanization, wealth, inequality, efficiency, risks, participation, elitism, power, dependence, welfare and mismanagement.

- Spain's position with respect to the European Union average on progress in scientific and technological research. The interviewees had to choose one of the following options: 1) Spain is in an advanced position; 2) Spain is at the same level; 3) Spain falls behind. - Level of the region of residence with respect to other Spanish regions regarding scientific and technological research. Options: 1) the region where I live is in an advanced position; 2) the region where I live is at the same level; 3) the region where I live is more advanced than some other, but not all, regions; 4) the region where I live falls behind all others.

- Actor mainly responsible for fostering scientific research and technological development. Options: 1) mainly, the European Union; 2) mainly, the Spanish government; 3) mainly, the regional government; 4) mainly, private corporations; 5) a shared responsibility of several of these actors.

- Evaluation of the degree of economic support given to scientific and technological research by the Spanish government, regional government and private corporations (separately). Options: 1) too many resources; 2) the necessary resources; 3) insufficient resources.

- The degree of agreement with the cutting back on funding in scientific and technological research due to a possible need by the government to reduce public expenditure. Options: 1) in favour; 2) against.

- The degree of agreement with various statements on the role of scientific knowledge in decision-making and public participation in issues related to science and technology (scale from 1 to 5). The statements were: 1) The work of scientists should be guided by those who pay for scientific research; 2) Scientists should decide the course and aim of their scientific activity; 3) If it has not been proven scientifically that new technologies can cause severe harm to humans or the environment, it is erroneous to impose restrictions on these technologies; 4) While the consequences of a new technology are not well known, action should be guided by caution and the technology's use should be controlled 
in order to protect health and the environment; 5) Scientific knowledge is the best basis for drawing up laws and regulations; 6) In the drawing up of laws and regulations, values and attitudes are as important as scientific knowledge; 7) Decisions on science and technology are best left with the experts; 8) The citizens should assume a more important role in decisions on science and technology.

- Spheres in which applied research should be given priority. The interviewees can choose two of the following options: IT and communications technologies, medicine and health, energy sources, food sciences, transport, protection of the environment, social and human sciences, aerospace technology, agriculture, public security and defence.

- Coverage given by the media to scientific information. Media included: daily press, free of charge press, radio, television, general interest weekly magazines. Possible answers: enough, not enough.

- Trust in media reporting on science and technology. Two options possible between: Internet, daily press, free of charge press, radio, television, general interest weekly magazines, science or technology weekly magazines.

- Trust in various institutions in dealing with issues related to science or technology (scale from 1 to 5). Institutions were: hospitals, professional associations, universities, public research institutions, political parties, labour unions, media, church, consumer associations, environmental associations, companies, and government and public administrations.

- Self-assessment of the level of scientific and technical education attained: very high, high, normal, low, very low.

- Areas where scientific-technical training has proven to be useful (scale from 1 to 5): profession, understanding of the world, relationships with other people, consumer and user behaviour, the formation of political and social opinions. Answers should be provided on a five-point scale, where 1 means almost useless, and 5 very useful.

- Socio-demographic variables: ideological self-classification, gender, marital status, formal education and religion.

\section{Statistical method and analysis}

Two types of analyses were carried out. First, a bivariate analysis of the relationship between the dependent variable and each of the independent variables using Pearson's Chi-square statistic. The value and significance of this test depends on sample size. In order to assure the findings' relevance, we also calculated Cramer's V test to obtain size effect estimations. This statistic offers values varying between 0 and 1 . A coefficient close to the value of 0 indicates that virtually no relationship exists, while a value close to 1 shows that the association is almost perfect. The purpose of the bivariate analysis is to have a general view of the association between the dependent variable and the factors that explain differences among supporters and non-supporters of public funding for scientific research.

Secondly, a multivariate analysis was applied to simultaneously analyse the effect of all the factors considered. Taking into account that our main aim is to identify the characteristics that will enable us to differentiate those subjects who are in favour of public expenditure for scientific research as compared to those who are not, a discriminant analysis with a stepwise method based on Wilks' Lambda test was performed. In stepwise estimation, each variable is entered in sequence and its value assessed. If adding the variable contributes to the model it is retained. All the variables in the model are then re-tested to see if they are still contributing to the success of the model. If not, they are removed (Brace et al., 2009). The stepwise method is a statistical procedure and it is the appropriate method when there are a significant number 
of independent variables and the most parsimonious model is sought (Brace et al., 2009). This statistical procedure could lead to the loss of predictive discrimination (Harrell, 2001). In addition, in linear models with large samples, of 1,000 cases or more (the size of the sample in this study is larger than 6,500), the statistical significance test is very sensitive, often indicating that almost any relationship is statistically significant (Hair et al., 1998). With large samples, the researcher must ensure that the criterion of practical significance is met along with statistical significance (Hair et al., 1998). Owing to both circumstances, beyond the model presented in this paper, we tested several other models which were discarded because of their lack of practical and conceptual significance, and statistical goodness-of-fit.

\section{Results}

\section{Bivariate analysis}

The strongest relationship can be observed between being in favour of the government increasing funding of science and technology and the declared interest in these activities. In this case, Cramer's V test has a value of 0.323 , a relatively high value compared to the coefficients usually obtained in opinion polls (Cramer's V around 0.1 ).

Less significant, but still important, are the following results:

Those who are in favour of an increase in funding of science and technology put a higher value on scientists and engineers, while those who do not mention this option tend to have a better opinion particularly of members of religious professions, sportsmen and sportswomen, lawyers, artists and journalists.

With respect to the relationship with the appraisal of science and technology, there is a well-defined tendency: those who support an increase in funding tend to answer affirmatively to the options that reflect what generally are supposed to constitute "positive" aspects of science and technology. The other group tends to answer affirmatively to the options that focus on "negative" consequences or aspects. Furthermore, the persons who think it's worthwhile to increase investment in science and technology tend to agree that the benefits of science and technology surpass any risks associated with both.

The two groups (those who support public funding of science and those who do not) also differ with respect to their general image of science. With regard to science, the former group makes an association with progress, well-being and above all, efficiency; to a greater extent, the second group makes an association with mismanagement, followed by dehumanization and lastly, risks. With regard to technology, the first group makes an association with welfare, progress and power; the second group with mismanagement and dehumanization (see also Todt and Luján, 2008).

In relation to the areas where priority should be given to applied research, there are only significant differences in the area of energy research and development, in that people in favour of increasing investment think that energy should be a prime objective $(\mathrm{V}=0.155)$.

A relevant fact in the use of scientific information is that supporters of increased funding of scientific and technological research are particularly trusting of the Internet $(\mathrm{V}=0.111)$, science magazines (0.183) and television (0.183) to obtain information on scientific topics. On the contrary, people who do not consider it necessary to increase funding tend to be among those who place the highest trust in institutions such as hospitals, professional institutions, the media, consumer associations and in particular, the Church.

Lastly, we need to point out that those who are in favour of greater funding of science and technology tend to think that the quality of scientific training received is high, whereas those who do not support greater funding think that the quality of training received is low. 
Furthermore, the first group states that scientific training has been personally useful in all facets of their lives; they particularly emphasized the professional aspect, followed by understanding of the world.

With relation to socio-demographic variables, it was found that people who defend a funding increase for research identify themselves with a left-wing political position, while the opposite happens with those who are against an increase in funding. Also, people with university studies (both in 3-year intermediate-level university studies, $\mathrm{V}=0.133$, and higher, 5 and more year university studies, $\mathrm{V}=0.140$ ) are more likely to be in favour of allocating more resources to science and technology than others, there being no significant difference in the other educational levels.

Summing up, these results are consistent with other perception studies and show the relation between, on the one hand, interest in and positive image of science and technology, and, on the other hand, a favourable attitude towards public funding, consistent with the hypothesis. Nevertheless, no relevant conclusions could be drawn from these results, not only due to the weak association between the dependent variable and most of the independent ones, but also because it is common that association and relationship among the variables cannot be well understood in isolation, meaning that significant bivariate associations could be spurious and their effect could be mediated by other more significant variables.

\section{Multivariate analysis}

A discriminant analysis has been used in this study because it helps to identify the characteristics that differentiate those who support public funding of science from those who do not. Furthermore it allows establishment of how many of these variables are necessary to attain the optimum allocation of interviewees to the two groups.

Table 1 shows the standardized coefficients of the discriminant function and the matrix data structure obtained when carrying out the discriminant analysis. Standardized coefficients

Table 1. Standardized coefficients and correlations

\begin{tabular}{lrr}
\hline Variable & Coefficient & Correlation (r) \\
\hline Science \& technology - interest in & 0.541 & 0.673 \\
S \& T offer the best knowledge about the world & 0.131 & 0.256 \\
S \& T contribute to a better environment & 0.214 & 0.233 \\
S \& T are not concerned with social needs & -0.172 & -0.136 \\
Same level of research in Spain and EU & -0.125 & -0.111 \\
Scientific and technological development are the responsibility of the set & 0.183 & 0.213 \\
of different governments (regional, Spanish, EU) & & -0.206 \\
The Central Government allocates sufficient resources to research & -0.195 & 0.038 \\
Companies allocate too many resources to research & 0.150 & 0.091 \\
IT and Communications should be a priority for applied research & 0.123 & 0.274 \\
The Energy sector should be a priority for applied research & 0.298 & 0.034 \\
The food sector should be a priority for applied research & 0.190 & 0.157 \\
Humanities and Social Sciences should be priorities for applied research & 0.237 & -0.456 \\
Level of scientific and technical information received & -0.231 & 0.329 \\
University studies - intermediate (3-year) & 0.184 & -0.188 \\
The extent to which Church and other religious officials are valued & -0.206 & -0.116 \\
Associates science with lack of control & -0.158 & 0.239 \\
Associates technology with power & 0.174 & 0.190 \\
Associates technology with dependency & 0.156 & \\
\hline
\end{tabular}

Source: Our elaboration (data: FECYT). Only those variables are shown which turn out to be discriminant. 
reflect the importance the variable has when predicting the group the interviewed person belongs to and represent the net contribution of each variable; i.e. the contribution of the variable without the rest of the variables included in the discriminant function.

The coefficients obtained indicate that the most important variable in terms of predicting the group to which the sample subjects belong is the stated interest in science and technology; followed by, at a considerable distance, the consideration of energy as a high priority area for applied research, the variable "Humanities and Social Sciences are a priority area for applied research," and the level of scientific and technical education received.

The centroids denote the average values for the groups in the discriminant function and enable us to establish which set of variables best characterize each group. For this purpose, we need to take into account the sign (positive or negative) of the coefficients and of the centroids. The variables that best define the supporters of increasing funding of science and technology are those that return a positive coefficient, given that the centroid for this group is positive (Centroid $=0.694)$. Non-supporters, instead, are defined by variables with negative coefficients (Centroid $=-0.274$ ).

From Table 1 and considering centroids, we can infer that individuals who think that government should increase funding for scientific and technical research tend to be interested in science and technology and consider that a) science and technology offer the best knowledge about the world; b) science and technology contribute to a better environment; c) scientific and technological development is a joint responsibility of the European Union, the central government, regional governments and private companies; d) companies allocate too many resources to research; e) IT and communication technologies, energy, the food industry and the human and social sciences are priorities for applied research; f) technology is associated with power and dependency. This group of people tends to have a middle-range university education (holding a diploma).

Those who are not in favour of increasing the government funding for scientific and technological research are defined by variables with a negative coefficient. These individuals believe the following: a) science and technology are not concerned with social needs; b) the level of scientific and technological research in Spain is equivalent to the European Union average; c) central government allocates sufficient resources to research; d) science is associated with being uncontrolled. They also believe that the level of science and technical education received is low or very low, and they value positively members of religious professions.

Table 1 also includes the matrix data structure or set of Pearson correlations between the discriminant variables and the discriminant function. These correlations measure the gross relationship of each variable with the discriminant function, i.e., without the influence of the other variables. We see that the variables most related to the discriminant function are stated interest in science and technology, level of scientific and technical education received and, in third place, middle-range university studies.

There are cases in which variables may show, at the same time, a very weak correlation with the discriminant function and relatively high standardized coefficients, indicating that these variables enable a clear differentiation between the two groups in spite of the weak relationship. In our case, the variables are: "companies allocate too many resources to research" and "the food sector should be a high priority for applied research." In fact none of these variables had a significant individual relationship with the dependent variable; nonetheless, in combination with the other variables, they contribute to the allocation of the subjects to one of the two groups.

There are other variables that have a high correlation with the discriminant function and a standardized coefficient which is sufficiently low so as not to enter the function; this means that in spite of the strong association between the variable and the function, they do not 
enable an adequate discrimination between the groups. Among all the variables which comply with this condition, the pair with highest correlations is: "scientific-technical training has been useful for my profession" $(\mathrm{r}=0.315)$ and "scientific-technical training has been useful in understanding the world" $(\mathrm{r}=0.304)$.

Information regarding the goodness-of-fit of the discriminant model is shown by the eigenvalue, canonical correlation and the value of Wilks' Lambda.

Eigenvalue (0.199) and canonical correlation (0.407) values indicate that there is considerable overlap between the two groups, in other words, that the discriminant variables do not adequately differentiate between those who choose the option of financing science and technology and those who do not. However, the significance level of Wilks' Lambda is less than 0.01 (Lambda $=0.834, p<0.001$ ), which enables us to state that the two groups have centroids that are separated enough to consider them as two distinct groups.

Once the discriminant function has been identified, it can be used to classify the same cases that were used to obtain it; thus the function can be used to assess the model's overall fit. The results are shown in Table 2.

The total number of correctly classified cases is high (79\%), but there are some notable differences in the cases that have been effectively classified in each group. In the group (which is also the biggest) of those in favour of increasing government funding in areas other than science and technology there is a correct classification of $93.9 \%$ of the cases; in the group of those in favour of increased government funding of scientific and technological research, correct classifications account for only $24.9 \%$. If we consider that we are comparing two groups, the random probability of correctly classifying them is $50 \%$ and hence the result is much worse than that expected by pure chance. It has been found that out of the 18 variables included in the discriminant function, 12 enable the classification of supporters of increasing funding of science and technology, while only six enable us to classify people belonging to the other group (Table 1). However, these 12 variables classify only $25 \%$ of those in favour of government funding of scientific and technological research, while the other six allow a correct classification of almost every subject in the group of non-supporters.

\section{Discussion}

Our results show that there is a direct relationship between the perception of science and the attitude towards its public funding. It is direct in the sense that those who have a positive perception of science tend to support its public funding. This is clearly reflected in the bivariate analysis.

Table 2. Results of the classification

\begin{tabular}{lllrrr}
\hline & & & \multicolumn{2}{c}{ Predicted group membership } \\
\cline { 3 - 5 } Increase in funding of S \& T & & Funding of science & $\begin{array}{c}\text { Funding of } \\
\text { another area }\end{array}$ & Total \\
\hline \multirow{2}{*}{ Actual group } & \multirow{2}{*}{ Number of cases } & Funding of science & 304 & 919 & 1223 \\
& & Funding of another area & 273 & 4167 & 4440 \\
& $\%$ & Funding of science & 24.9 & 75.1 & 100 \\
& & Funding of another area & 6.1 & 93.9 & 100 \\
\hline
\end{tabular}

Correctly classified: $79 \%$ of the cases.

Source: Our elaboration (data: FECYT). 
The variable most related to the lack of support for public funding is the level of scientific and technical education received (Coefficient $=-0.231, r=-0.456$; the negative sign of the correlation indicates an inverse relationship ${ }^{3}$ ). This result could be taken as evidence for the argument in favour of the cognitive deficit model but we must bear in mind that the question asked refers to the self-perceived level of science and technology education. However, if we consider the actual level of education (from the data relative to the individual's received education), we find that the only formal education level that is statistically significant is the category of intermediate (3-years) university studies. The variable with the biggest weighting in characterizing this group is the perception of a low educational level in science and technology. This suggests that behind this answer there is the feeling of limited knowledge on science and technology among the interviewees of this group. Another relevant variable identifying those in this group is the extent to which religious authorities are valued, although the Pearson correlation value is small.

The discriminant function does not enable us to adequately identify the characteristics which define those in favour of public funding (we should remember that only $24.9 \%$ of cases are adequately classified). The variables of the survey used in our analysis do not allow us to adequately differentiate between the two groups. This indicates an overlap between the groups; in other words, the individuals, independently of their attitude towards funding, respond in a similar fashion to most of the questions of the survey. Even more, no significant influence of socio-demographic variables has been identified.

The results of this analysis reveal that the general perception of science, as may be determined by the usual questionnaires in public perception studies, has limited predictive value with respect to the support of public funding of science. In the interpretation of these findings, we have considered three main points.

First, although only $20 \%$ are in favour of public funding for science, the remaining $80 \%$ simply choose other areas because they are thought to be more important, in other words, they are not against public funding of scientific and technological research. Hence, they are nonsupporters, not detractors.

Second, the multivariate analysis shows that the key variable is interest in science and technology. In other words, the interest reflects a positive global opinion that gathers up the information revealed by other, more specific variables (questions). Once this variable on interest in science and technology is included in the model, the others can be considered to be incidental or indeed superfluous.

Third, the discriminant analysis leaves out many of the variables related to the public perception of science that in the bivariate analysis correlate with a positive disposition towards public financing of science and technology (some of which, furthermore, intuitively would seem related to the attitude towards funding). It would seem that the variables commonly applied in public perception studies have limited predictive value with respect to the attitude towards public funding of scientific research, or the differentiation between those in favour and those not in favour of public funding.

\section{Conclusions}

The multivariate analysis (Wilks' Lambda) applied in this study produces results that are significantly different from the results given by the bivariate analysis. Whereas the bivariate analysis allows the identifying of positive correlations with respect to a number of variables, the multivariate analysis shows that most of these variables do not have discriminant capacity with respect to the attitude to public funding of science and technology. 
We can draw a substantive as well as a methodological conclusion: on the one hand, based on the data from a standard survey, it turns out to be difficult to predict an individual's attitude towards financing. On the other hand, the combination of different statistical techniques (as in this case a standard statistical bivariate analysis complemented by the multivariate/Wilks' Lambda analysis) allows us to qualify and put into context the survey results. Multivariate analysis complements the bivariate one.

On the basis of these conclusions we can suggest two lines for future development of this area of research. One is to identify variables related to the public understanding of science that are useful for characterizing the group of people who clearly support public funding of science. Given that those variables that are generally used in public perception studies have shown little predictive power, future research could identify variables with more predictive power.

The second suggestion concerns the questionnaires used in studies of public perception of science. If we accept that the support for public funding of scientific research is an important feature of the perception of science, the degree to which a variable contributes to identify the group of people in favour of public funding can be used as a criterion to select some of the questions to be included in future questionnaires.

\section{Acknowledgements}

The work presented in this paper was carried out as part of the projects El principio de precaución en la evaluación de riesgos (HUM2006-12284/FISO) and Concepto y dimensiones de la cultura cientifica (FFI2008-06054) from the Spanish Ministry of Science and Innovation and co-financed with FEDER funds from the European Commission. The authors are grateful to the FECYT for the data from the survey.

\section{Notes}

1 Including those who did not choose any of the options $(0.5 \%$ of the total), or those who chose the options "don't know" (3.3\% of the sample) or "doesn't answer" $(0.3 \%)$.

2 The main findings of this survey and the exact wording of the questions in Spanish can be found at FECYT's website, http://www.fecyt.es

3 The level of scientific and technical education received ranges from 1 - Very high, to 5 - Very low; the variable for the support of public funding has been codified as 0 (It should not be funded) and 1 (It should be funded). Hence the inverse relationship indicates that supporters of public funding tend to affirm that the level of scientific and technical education received is high, while those who are not in favour of public funding tend to affirm that the level of education received is low.

\section{References}

Allum, N., Sturgis, P., Tabourazi, D. and Brunton-Smith, I. (2008) "Science Knowledge and Attitudes across Cultures: A Meta-analysis," Public Understanding of Science 17: 35-54.

Bauer, M. (2002) "Controversial Medical and Agri-food Biotechnology," Public Understanding of Science 11: 93-111.

Brace, N., Kemp, R. and Snelgar, R. (2009) SPSS for Psychologists, 4th edn. Mahwah, NJ: Palgrave Macmillan.

Godin, B. (2002) “Outline for a History of Science Measurement," Science, Technology, \& Human Values 27: 3-27.

Hair, J.F., Anderson, R.E., Tatham, R.L. and Black, W.C. (1998) Multivariate Data Analysis, 5th edn. Englewood Cliffs, NJ: Prentice-Hall.

Harrell, Jr., F.E. (2001) Regression Modeling Strategies: With Applications to Linear Models, Logistic Regression, and Survival Analysis. New York: Springer.

Irwin, A. and Michael, M. (2003) Science, Social Theory and Public Knowledge. Maidenhead: Open University Press.

Irwin, A. and Wynne, B. (1996) Misunderstanding Science? The Public Reconstruction of Science and Technology. Cambridge: Cambridge University Press. 
Kallerud, E. and Ramberg, I. (2002) "The Order of Discourse in Surveys of Public Understanding of Science," Public Understanding of Science 11(3): 213-24.

López Cerezo, J.A. and Cámara, M. (2007) "Scientific Culture and Social Appropriation of Science," Social Epistemology 21: 69-81.

Luján, J.L. and Todt, O. (2000) "Perceptions, Attitudes and Ethical Valuations: The Ambivalence of the Public Image of Biotechnology in Spain," Public Understanding of Science 9: 383-92.

Michael, M. (1992) "Lay Discourses of Science: Science-in-General, Science-in-Particular and Self," Science, Technology, \& Human Values 17: 313-33.

Michael, M. (2002) "Comprehension, Apprehension, Prehension: Heterogeneity and the Public Understanding of Science," Science, Technology, \& Human Values 27(3): 357-8.

Millar, R. and Wynne, B. (1988) "Public Understanding of Science: From Contents to Processes," International Journal of Science Education 10(4): 388-98.

Miller, J.D. (2004) "Public Understanding of, and Attitudes toward, Scientific Research: What We Know and What We Need to Know," Public Understanding of Science 13(3): 273-94.

Pardo, R. and Calvo, F. (2002) "Attitudes toward Science among the European Public: A Methodological Analysis," Public Understanding of Science 11: 155-95.

Pardo, R. and Calvo, F. (2004) "The Cognitive Dimensions of Public Perceptions of Science: Methodological Issues," Public Understanding of Science 13: 203-27.

Sturgis, P.J. and Allum, N.C. (2004) "Science in Society: Re-evaluating the Deficit Model of Public Attitudes," Public Understanding of Science 13(1): 55-74.

Todt, O. and Luján, J.L. (2008) "A New Social Contract for Technology? On the Policy Dynamics of Uncertainty," Journal of Risk Research 11: 509-23.

Todt, O., Muñoz, E. and Plaza, M. (2007) "Food Safety Governance and Social Learning," Food Control 18: 834-41.

Todt, O., Muñoz, E., González, M., Ponce, G. and Estévez, B. (2009) "Consumer Attitudes and the Governance of Food Safety," Public Understanding of Science 18(1): 103-14.

\section{Authors}

Ana Muñoz van den Eynde is researcher at the Research Unit on Scientific Culture of CIEMAT (Centro de Investigaciones Energéticas, Medioambientales y Tecnológicas), Spain. Her research interests are in the public perception of science and environmental issues. Correspondence: Research Unit on Scientific Culture, Centro de Investigaciones Energéticas, Medioambientales y Tecnológicas (CIEMAT), Avda Complutense, 22, 28040 Madrid, Spain; e-mail: ana.munoz@ciemat.es

Carolina Moreno is Head of the Department of Theory of Language and Communication at the University of Valencia (Spain), and a member of the Research Unit on Scientific Culture of CIEMAT. She is professor of science communication and her research focuses on content analysis of media reports related to science and technology.

José Luis Luján is full professor of philosophy of science at the University of the Balearic Islands (Spain), and a member of the Research Unit on Scientific Culture of CIEMAT. His research interests are in the role of scientific knowledge in science and technology regulation. 